\title{
Le show d'Angèle
}

\author{
Claudine Potvin
}

\section{Angel's Show}

Viewing is the privileged act of culture's legitimated subjects and pornography makes explicit the gender specificity of the dominant social relations of seeing and being seen. The pornographic eye not only objectifies its subject, dispossesses her of her body, and obliges her to find pleasure in the fantasy of a man, but also naturalizes the one-way gaze of man at woman by forbudding her to exercise a gaze of her own. This text stages the scene of production of a pornographic film. It interrupts the film's system of looking by interweaving the perspective of the woman violated in/by the film with the perspective of a woman who returns the gaze of the camera by reviewing and rewuriting the scene.

\section{La mort pour moi est accoutumance}

et elle se présente sous forme de strangulation ou d'étouffement avec un objet mou sur ma bouche jusqu'à ce que mon souffle cesse de se produire. (Louky Bersianik, La main tranchante du symbole)

The woman zoo extends beyond the metal slots and shutters of the peep-show. The cultural space of women, captured and framed in images, is one big Show World. There remains but one kind of look: looking at Man gazing at woman, one-way. (Susanne Kappeler, The Pornography of Representation)

Angèle s'étira le cou pour voir où en était la caméra. L'homme n'avait pas fini de la violer que déjà la fatigue la gagnait. Faire semblant de se révolter ou faire semblant d'aimer ça du pareil au même. Dans le fantasme de l'homme, il fallait qu'elle y trouve son plaisir, son supplément. Lire sur sa peau les marques d'une résistance lointaine, passée, la feinte. 
L'arrogance du projecteur affiche les cernes creusés de son jeune sexe plein jour les corps se frottent contre la barre transversale du lit défait l'éclairage bute sur l'obscur désir de se refaire une vie gros plan sur les lèvres rosées elle a de plus en plus soif et la séance d'essayage s'éternise se mettre le corps des autres sur le dos pour voir s'ils s'ajustent bien aux hanches au buste à la taille trop longs trop courts les tissus veloutés des peaux noires sur blanches ne glissent pas sur la chair irritée à recommencer autant de scènes perdues dans sa mémoire fondue enchaînée sur une suite de bras pendus les uns sur les autres.

Angèle fait du cinéma depuis l'âge de neuf ans. Elle sait (elle savait) que seul le regard compte. Du fond de la salle, il la contemple dur rigide fort aigle implacable sous l'aile couronnée d'une tête blanche en désordre.

Quand ils l'enculent, ça ne fait presque plus mal. Une moue au coin de la bouche une grande respiration et le tour est joué. Il y a des années que les mots ne la font plus sourire ou rougir ni les gestes s'approprier la langue de l'autre en faire à sa guise revenait à se placer dans son objectif sans appui. Angèle ne pense pas échapper à l'ordre des prises de vue se contente de manger sa crème glacée à la vanille que Pierre vient de lui apporter. Il fait chaud dans ce garage humide portes verrouillées au cas où le vent du dehors s'y infiltrerait la machine à fabriquer le vent joue sans cesse dans le cou de l'ange se balade sur ses petits seins étonnés retrousse sa chevelure rousse pour l'effet pendant que des mains l'attirent vers le décor fabriqué pour la scène initiale. Angèle adore la machine à vent et se laisse faire une sorte $d^{\prime}$ amour improvisé pour la circonstance. L'amour c'est l'oeil de l'autre c'est la voix nasillarde du directeur qui orchestre le jeu son jeu les mouvements la sensation d'un doigt qui pince d'un dos plié sur son ventre la rage des hommes et des femmes en train de sucer son âme l'amour c'est la solitude la faim des autres la trace du stylo entre l'aîne et la fente la piqûre d'héroïne. The show must go on, (se) répète-t-elle.

Pour que l'enfant accepte d'ouvrir les cuisses, on lui donne des friandises aux fraises. Il n'y a que l'odeur rouge des fraises pour la convaincre de s'abandonner. Au fond du studio l'oeil avisé du spectateur frémit de la voir si fraîche sans retenue attentive au seul plaisir de l'absence la bouche de l'intrus fouille ce sexe imberbe tourne de l'oeil sous le regard obsédé obsédant des figurants vivre comme si tout celà ne la regardait pas. 


\section{Le show d'Angèle · 27}

Elle n'est certes pas (toute) là. Elle (re)entre (re)joue le corps polymorphe re(de)vient image.

The woman, object/victim par excellence in the structure of representation, is now being invited to join the readership and the spectators as a "voluntary audience" of the great cultural fiction - the patriarchy represented - now that the structure of representation, the scenario in representation, have been defined and confined to the transitive plot, the coercive imperative, now that the (male) gendered act of viewing and of reading has been naturalized. (Susanne Kappeler, The Pornography of Representation)

Un malaise se glisse pourtant sous la manipulation. Quelque chose lui échappe. Si elle n'a déjà plus de corps, si le sens dérive au-delà de l'instantané, si ses yeux s'apprêtent à la repeindre en bleu, si la pose efface les rides à venir, si elle est presque morte sous l'enveloppe, un reste de poudre blanche sur la coiffeuse se perfile encore dans son champ de vision, un mégot mal éteint dans la chambre de bains et le parfum de sa mère. Effacée. Délavée sous la pluie d'avril. Un jour, sa mère a tourné le coin de la rue et elle ne l'a plus jamais revue. Ne lui en est resté que des robes en chiffon dont elle se pare désormais dont elle se défait sous le rire de ses strip-teases enfantins. Pas de strip-tease réel pour le pornographe. Pas de découpage ni de dévoilement. La victime est déjà toute nue. Le récit s'engage sur une piste dérapante les courbes d'Angèle les voiles cachés glissent pentes perfides mauvais penchants.

Dans ses rêves Angèle se déshabille toujours pour sa mère qui n'en revient pas Pierre crie trop fort et sa vie tourne autour d'un plateau dérisoire ne jouera jamais sa véritable histoire celle d'une chute le mépris s'installe entre chaque prise sa peau change d'odeur lui la toise de plus haut depuis qu'il assiste aux répétitions elle pressent la défaite objet rare de musée imaginaire en montre toile exposée devant les passants qui défilent détachés bien installés dans leurs souliers vernis hors du cadre en dehors de toute fiction qui la nomme mannequin de cire voyeurs solitaires d'un spectacle interdit identification non permise la pellicule circule se coupe ne filtre que de faux désirs fondent sous les réflecteurs mise en scène douteuse.

Angèle circule tard dans les rues de la ville. Le sommeil la rejoint aux petites heures du matin accoudé sur une bouche de chaleur. Refuse de jouir. 
Entre chaque séance, Angel, ainsi renommée par le caméraman argentin, se déguise et joue à la madame avec Colette qui traîne toujours entre les fils. Elles se coiffent devant le grand miroir du fond, se relèvent les cheveux en chignons pour avoir l'air plus vielles, déambulent à talons hauts, trébuchent sur les coussins, se remettent du rouge sur les joues, font semblant de se donner, se refont une beauté pour l'indifférence des gars, s'étendent dans toute la perversité de leurs regards et puis, désabusées, allument une cigarette.

Mirna interrompt le récit. Penchée sur le texte. Le corps d'Angèle éclate puzzle Mirna n'arrive pas ne veut pas replacer la lettre d'origine le sujet pornographique s'impose détruit l'objectif de la narration. Mirna s'étire les bras et se masse légèrement le cou pour relâcher la tension. Elle rallume la cigarette éteinte et va s'installer devant le téléviseur. L'écran lui renvoie l'image d'une réalité truquée, arrangée d'avance par le gars des vues. Impossibilité de se placer en dehors de ou derrière, de démonter la (re)présentation. Le petit écran ne retient que la vision avec/le reportage en appelle à l'autobiographie le do(cu)men/taire t'es l'objet. Mirna interroge la texture porphyrö̈de de son obscène histoire d'ange. Le cliché de l'ange déchu, songe-t-elle. Elle éteint et remet Ella Fitzgerald pour la millième fois. La mémoire des notes infiltre lascivement la lumière opaque de ses cernes. Elle renifle son cognac et laisse glisser telle une chatte imperceptible une main gauche le long de sa cuisse. Pour ne pas interrompre le charme de l'écriture, poursuit-elle.

Angèle et Colette jouent avec leurs Barbies, inventent un conte de fée autour du corps gelé de leurs poupeés aux longs cheveux satinés, vêtent et revêtent les minces figurines de roses éclatants, parlent de fuites, de la venue de Ken, de décors au fond des boîtes. Lasses, elles les abandonnent toutes nues, sans sexe, inutiles, au milieu d'une scène.

Mirna ne sait trop ce qu'elle en pense. Trop jeunes. Wild at heart. Se sent observée par dessus l'épaule la nuit suinte sous les doigts se compose une attitude imagine le corps fragile de la victime à la renverse un peu perdu sous les oreillers une sorte de dérive contrôlée comme dans un film d'horreur entre le ravissement et la peur lit que " ... voyeurism, fetishism, or the imaginary signifier ... are directly implicated in a discourse which circumscribes woman in the sexual, binds her (in) sexuality, makes her the absolute representation, the phallic 
scenario. It is then the case that the ideological effects produced in and by those concepts, that discourse, perform, as dominant cinema does, a political function in the service of cultural domination including, but not limited to, the sexual exploitation of women and the repression or containment of female sexuality." (Teresa de Lauretis, Alice does- $\left.n^{\prime} t\right)$. Mirna auteure spectatrice narratrice narrataire lectrice se noie dans l'ambivalence de son personnage voudrait faire un film là-dessus mais le scénario lui échappe le sujet aussi certains films sont lourds à visionner pornographe auteure d'un traité sur la prostitution.

Angèle / Angel reprennent la pose s'installent en filigrane pour peu qu'on leur demande, rampent complaisamment sous les feux font durer le plaisir de l'autre sous la tension de la bouche resserrée sur le membre qui s'accroche the prick of the object violence du flash éjaculatoire perpétuer les mythes se gargariser d'érotismes humiliants sous l'angle d'un désir féminin d'enfante suraimée, l'orgasme ne reconnaît plus la coulée secrète la joie l'attente la délicatesse se lèvent d'un trait repoussent la pesanteur de l'autre s'interdisent la colère fixent la caméra se

Mirna imagine qu'Angèle crie (castration / frustration). Le stade du cri. Elle avait voulu Angèle passive, victime, innocente. Par quel obscur déplacement cherche-t-elle dorénavant la trace d'une enfant rebelle? La plainte nouvellement surgie de la chair d'Angèle ravive une jouissance ancienne les passions malsaines de l'enfance l'obsession du cliché marquée au vif par cette stupide histoire refoulée loin derrière les gestes de l'ennui saisie du quotidien fenêtres closes de sexes dits puérils vicieux descentes de lit pour petites filles seulement. Du côté de Sade, Barthes raconte que "Le cri est la marque de la victime: c'est parce qu' elle choisit de crier, qu' elle se constitue victime; si, sous la même vexation, elle en venait à jouir, elle cesserait d'être victime, se tranformerait en libertin: crier/décharger, ce paradigme est le départ du choix, c'est-à-dire du sens sadien." (Roland Barthes, Sade, Fourier, Loyola). Mirna cherche son cri fétiche étranglé dans sa gorge au-delà de l'insignifiante langue en mutation interroge le libertinage comme forme d'affirmation le jet comme soupçon le flot déversé lancé hors de soi le vagin d'Angèle resté sec alors même que les parois désertiques du langage se mouillent en silence nomment prudemment la peur inscrite dans l'oeil de pierre fixations obscènes masturbation/visionement des hommes libertins n'en finissent plus de venir 
sur la page contrôlent dominent font souffrir au lieu de souffrir. Mais $l^{\prime}$ ange de Mirna ne devait rien savoir de tout cela. Son ange roux s'envole sans qu'elle n'y puisse rien.

Angèle se débauche un peu trop au goût de son père danse sur une corde raide qui ne lui appartient plus s'initie au jeu du chat et lui sourie cochonne s'invente une parabole un théâtre fermé pour adultes seulement dirige la lentille close-up sur sa vulve insoumise effet loupe, murmure-t-elle, effet cumul autant d'ouvertures qu'on rêve de déchirer de pénétrer de trans/percer de transposer pièce en morceaux détachés zoom sur le sein gauche le cul droit l'anus à perforer un corps à dévorer cannibalisme de sursis.

Angèle/Angel entendent des voix dans les coulisses s'approchent du mur du son acoustique superflue la chambre en aérosol suffoque Angèle sent la main de son père envahir l'écran se poser sur elle papillon glacé sur sa gorge blanche you are the breathless hush of evening that trembles on the brink of a lovely song / you are the angel glow that lights a star I the dearest things I know are what you are la violence du coup suit on tourne la séquence père/fille l'orgie se résorbe dans le sang trop occupé à se répandre dans les trous d'une chaîne de montage.

Art sans prétention. Mirna clôt le texte. Vider le sac, le surplus, l'épisodique. Le sexe mou de l'homme repose sur une cuisse placide en attente de l'autre ne viendra plus l'exécution d'Angèle agresse Mirna auto-graphie du récit au comble à la limite de incestueuse et parricide, Mirna reçoit la geste d'Angèle comme un coup de fouet les traces rouges sur sa joue inscrivant une certaine écriture cinématographique au lieu précis de l'exploit(ation). 